\title{
Les recommandations de la Commission internationale de protection radiologique, $\operatorname{I977}\left({ }^{*}\right)$ \\ C. G. STEWART $(* *)$
}

\begin{abstract}
RÉSUMÉ
Après un bref rappel historique, l'auteur expose les nouvelles recommandations de la C.I.P.R. (publication CIPR 26) et leurs fondements.
\end{abstract}

\section{ABSTRACT}

After a short historical review, the new recommendations of the I.C.R.P. (ICRP publication 26) and their basic concepts are explained.

Trois mois [1] après la découverte des rayons $\mathrm{X}$ par RoenTGEN [2] en 1895, les expérimentateurs avaient déjà pris conscience de la nécessité d'une protection contre les effets nocifs des rayonnements ionisants. Dans les décades qui suivirent, les rayons $\mathrm{X}$ et les rayonnements émis par les éléments radioactifs découverts par BECQUEREL [3] en 1896 entraînèrent chez les utilisateurs un taux de lésions tel qu'en 1922, Ledoux-LeBARD [4], à Paris, estimait à plus de cent le nombre des pionniers de la radiologie morts de cancer résultant de leur profession. Ce fut dans ce contexte que le second Congrès international de Radiologie, réuni à Stockholm en 1928, créa pour la première fois un Comité International auquel fut confiée la tâche de formuler des recommandations pour la protection des membres du Congrès dans la pratique de leur Art et de leur Science. Ce comité du Congrès vécut jusqu'à la $2^{\circ}$ Guerre mondiale, émettant des recommandations pour la protection contre les effets

(*) Communication présentée lors du $4^{\mathrm{e}}$ Congrès international de l'Association internationale de Radioprotection (A.I.R.P.), Paris, 24-30 avril 1977.

(**) Atomic energy of Canada Ltd, Chalk River nuclear laboratories, Chalk River, Ontario, Canada.

RADIOPROTECTION, VOL. $13-\mathrm{N}^{\circ} 4$ 
nocifs des rayons $\mathrm{X}$ et des rayonnements du radium. Reconstitué en 1950 sous un nouveau nom, il devint la Commission Internationale de Protection Radiologique (C.I.P.R.).

En 1950, le besoin de protection contre les rayonnements avait pris des dimensions entièrement nouvelles. La Commission nouvellement reconstituée reconnut que le "Comité National de Protection contre les Radiations" des États-Unis (N.C.R.P.) avait réuni, entre 1946 et 1950, une somme considérable d'informations en raison des connaissances acquises dans les domaines de l'énergie nucléaire et de la radiobiologie. Ces informations concernaient les dangers associés non seulement à l'exposition à des sources externes de haute énergie, mais aussi bien à un mode d'irradiation qui devait jouer un rôle beaucoup plus important dans le monde d'après guerre : celui qui résulte du dépôt dans l'organisme humain de substances radioactives. Lors de la rédaction de ses premières recommandations en 1950 [5], la C.I.P.R. reçut les conseils d'experts du Canada, de France, d'Allemagne, de Grande-Bretagne, de Suède, tout autant que de personnes associées au N.C.P.R. américain. Pour la première fois, elle émit des recommandations concernant les quantités incorporées et les taux d'incorporation de ces radionucléides - une douzaine au total. A cette époque également, la Commission reconnut pour la première fois qu'en plus de la radiologie médicale elle avait un besoin impérieux de conseils dans le domaine de la recherche scientifique. A cette fin, lors de sa session de 1950, elle créa en son sein une structure de comités consultatifs permanents, composés d'experts chargés de donner leur avis sur les questions des doses reçues à partir de sources externes et de sources internes. Encore aujourd'hui, avec quelques modifications, ces comités continuent à conseiller la Commission. Cet événement, rétrospectivement, représente la première rupture de la Commission avec son passé.

Avant la $2^{\mathrm{e}}$ Guerre mondiale, ses conseils s'étaient presque exclusivement basés sur l'expérience acquise par les utilisateurs de rayons $\mathrm{X}$ et de radium à des fins médicales. Au cours des années 50 et 60 , l'élaboration des recommandations de la Commission bénéficia des apports sans cesse plus importants non seulement de la médecine et de la physique, mais aussi de la radiobiologie, de la biochimie, de la génétique, de l'épidémiologie - pour ne citer que quelques-unes des disciplines qui ont contribué aux recommandations générales de la Commission publiées successivement en 1954, 1958, 1962 et 1965 [6-9].

Durant cette période, les notions issues de la radiologie clinique se virent également modifiées. Nous apprîmes qu'il n'y avait pas de dose "tolérable " pour l'ensemble des réponses biologiques aux rayonnements ionisants. Tandis que nous trouvions qu'un petit nombre de réponses était caractérisé par un seuil, l'effet augmentant ensuite en même temps que la dose, les effets que nous pressentions comme de plus en plus importants - les dommages génétiques et l'induction de cancers - n'étaient apparemment pas caractérisés de façon générale par un seuil; pour ces réponses, il s'agissait plutôt d'une affaire de «tout ou rien », dont la probabilité d'apparition diminuait avec la dose mais dont l'augmentation de fréquence se manifestait probablement jusqu'aux doses les plus faibles. Au cours de cette période, la Commission commença, 
en matière de protection, à évoluer progressivement d'une position basée principalement sur l'expérience des rayons $\mathrm{X}$ et sur les concepts de seuils et de doses tolérables, jusqu'à une philosophie du risque [10]; il fut, alors, facile de franchir un nouveau pas en considérant la grandeur des risques liés à des niveaux particuliers de doses en corrélation avec les avantages procurés par les pratiques qui entraînent ces niveaux de dose.

Ce furent des considérations de cette nature qui conduisirent aux nouvelles Recommandations générales de la C.I.P.R. La Commission raisonna de la façon suivante : dans l'idéal, à un niveau de risque devrait être associé les avantages qui résultent du risque encouru. Cependant, après avoir reçu l'avis des groupes de travail, la Commission estima que la généralisation d'analyses coût-avantage de ce genre, dans le domaine de l'utilisation des rayonnements ionisants, n'était pratiquement pas encore possible. Dans une première étape, elle entreprit donc de concevoir un système de limites de dose basé sur le principe de l'égalité du détriment (risque) pour chaque limite de dose. Pour ce faire, il fallait relier des niveaux de risque à des limites de dose par l'intermédiaire de considérations quantitatives sur les relations dose-effet. Il fallait, ensuite, choisir un critère du détriment et, enfin, un critère d'acceptabilité du détriment. Bien que l'invalidité, la maladie, la mort entrent, à un titre quelconque, dans toute considération générale du détriment, on estima qu'au départ, tout au moins, on pouvait choisir comme mesure du détriment le taux maximal estimé de mortalité. Ceci nous parut justifié pour plusieurs raisons : les invalidités dues aux rayonnements ionisants sont susceptibles de présenter une proportion plus faible de cas non-mortels par rapport aux cas mortels que dans la plupart des autres professions; si l'on devait maintenir l'exposition moyenne aux rayonnements à un niveau de sécurité élevé en ce qui concerne la conséquence "décès", alors les travailleurs affectés à des travaux sous rayonnement seraient à un niveau de sécurité bien plus élevé en ce qui concerne les autres incapacités professionnelles que ceux de la plupart des autres industries. Dans un but d'analyse, il fut alors décidé que le risque de décès associé aux limites de dose serait de l'ordre des risques associés aux activités industrielles ou de laboratoires les plus sûres. Enfin - et c'est une démarche nouvelle de la part de la Commission - il fut décidé qu'il faudrait prendre en considération la fréquence du dommage génétique induit par les rayonnements et manifesté dans les deux premières générations d'individus exposés pour déterminer l'acceptabilité de l'exposition de ces individus et, par conséquent, les limites de dose pour l'individu. Il fut admis que le dommage génétique apparaissant dans les générations ultérieures serait intégré au lot général des mutations de la population.

On sait qu'en général, dans l'industrie, des sous-groupes de travailleurs d'une branche particulière sont soumis à des risques plus élevés que la moyenne; en d'autres termes, à l'intérieur d'une même branche, il y a une distribution de la fréquence des risques qui peut se caractériser par une valeur moyenne. Dans certaines situations où l'on dispose d'informations sur les risques de sous-groupes particuliers, il semblerait que ces risques soient 5 fois supérieurs à la moyenne [12-15]. Les sous-groupes, eux-mêmes, ne sont pas entièrement homogènes, en sorte que, selon toute vraisemblance, certains

voL. $13-\mathrm{N}^{\circ} 4$ 
risques individuels dans ces branches diffèrent de la moyenne par au moins un ordre de grandeur. En fait, l'expérience montre que, dans la plupart des installations affectées à des travaux sous rayonnements, les doses annuelles moyennes des travailleurs surveillés sont généralement inférieures au dixième des doses enregistrées pour les individus les plus exposés. Dans des branches particulières comprenant un nombre élevé de travailleurs auxquelles s'appliquent les recommandations de la Commission, la distribution en fréquence des doses reçues est bien représentée par une fonction de distribution lognormale caractérisée par un écart-type géométrique de 2,5 ou plus. Les limites de dose sont rarement dépassées, la fréquence étant de l'ordre de $10^{-3}$. Si l'on suppose que cette fréquence correspond aux limites de dose dans la distribution, alors le rapport de la limite de dose à la dose moyenne du groupe sera au moins de dix (en fait, avec un écart-type géométrique de 2,5, le rapport à la moyenne géométrique serait $\sim 17$ ).

$\mathrm{Si}$ nous désirons donner au risque une valeur quantitative - or, nous ne mesurons pas directement le risque, mais nous estimons plutôt la dose de rayonnement reçue - alors, comme je l'ai indiqué, nous devons connaître les relations "dose/réponse ». Il n'y a aucune raison a priori pour que les fonctions mathématiques reliant la réponse à la dose soient nécessairement les mêmes pour différentes réponses, fût-ce dans la même espèce. Nous savons depuis longtemps que certaines réponses sont caractérisées par des seuils et, dans ce cas, en général, plus la dose augmente, plus la réponse s'accentue. Dans les nouvelles recommandations de la Commission, nous appelons "non-stochastiques » les réponses de ce genre. Pour empêcher qu'elles ne se produisent, il suffit de s'assurer que les limites de dose correspondantes sont inférieures au seuil où se manifeste la réponse. Nous appelons "effets stochastiques » les autres réponses à l'irradiation : essentiellement de nature "tout ou rien », ils surviennent généralement sans qu'un seuil soit mis en évidence et leur fréquence augmente avec la dose; nous savons, actuellement, que les plus importants de ces effets stochastiques sont l'induction de cancer ou de dommages génétiques.

Jusqu'ici, la connaissance de la forme exacte des relations « dose/réponse » chez les populations humaines est trop limitée pour permettre une prévision sûre des courbes et de leurs pentes aux faibles doses et aux faibles débits de dose. Puisque nos connaissances sur les relations «dose/réponse » proviennent, en grande partie, de situations où dose et débit de dose, à la fois, étaient élevés par rapport à la zone couverte par les limites de doses recommandées, la Commission demeure d'avis que la fréquence réelle des effets par unité de dose (pente) devra être plus faible à la suite d'une exposition à des faibles doses ou à des doses délivrées à de faibles débits de dose. Il peut donc être judicieux, dans certains cas, de réduire ces estimations d'un facteur qui prenne en considération une différence probable de risque. Sur la base de toutes les données disponibles, la Commission a ainsi choisi pour le risque des valeurs par unité de dose (que je discuterai brièvement) qui puissent être utilisées en pratique pour la radioprotection. Par ailleurs, une hypothèse fondamentale, de façon générale prudente, demeure toujours sous-jacente aux recommandations de la Commission : pour les effets stochastiques 
et dans la limite des conditions d'exposition que l'on rencontre habituellement pour les travaux sous rayonnements, il y a une relation linéaire sans seuil entre la dose et la probabilité de réponse à cette dose.

Dans le passé, la Commission avait énoncé que lorsque plus d'un organe ou d'un tissu était exposé, on pouvait considérer comme critique un organe ou un tissu particulier, en raison de la dose reçue, ou de sa sensibilité aux rayonnements, ou de son importance pour la santé; la limitation de dose pour l'individu était alors déterminée par la limite d'équivalent de dose pour ce tissu. Ce concept d'organe ou de tissu critique interdit de faire la somme des détriments en fonction de la radiosensibilité relative des tissus irradiés. La Commission recommande, désormais, une procédure qui tienne compte du risque total résultant de l'exposition de tous les tissus irradiés.

Tout d'abord, les tissus considérés comme suffisamment radiosensibles pour mériter une évaluation individuelle du risque stochastique associé à leur irradiation sont les suivants :

\begin{tabular}{|c|c|c|}
\hline & Risque par Sievert & Risque relatif $w_{t}$ \\
\hline Gonades............ & $4 \times 10^{-3}$ & 0,25 \\
\hline Sein.$\ldots \ldots \ldots \ldots \ldots$ & $2,5 \times 10^{-3}$ & 0,15 \\
\hline Moelle rouge des os..... & $2 \times 10^{-3}$ & 0,12 \\
\hline Poumon.............. & $2 \times 10^{-3}$ & 0,12 \\
\hline Thyroïde............. & $5 \times 10^{-4}$ & 0,03 \\
\hline 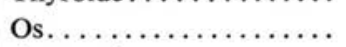 & $5 \times 10^{-4}$ & 0,03 \\
\hline Autres tissus. . . . . . . . & $5 \times 10^{-3}$ & 0,30 \\
\hline
\end{tabular}

Après une étude des données disponibles, la Commission a alors affecté à chacun de ces tissus un risque par unité d'équivalent de dose reçu (tableau ci-dessus). On peut faire quelques commentaires sur leur choix et les réponses particulières aux radiations pour lesquelles le risque a été estimé.

Les effets nocifs d'une irradiation des gonades humaines comprennent : l'induction de cancer, la diminution de la fécondité, et des effets héréditaires chez les descendants des individus irradiés. Les gonades semblent relativement peu sensibles à l'induction de cancers et, en comparaison de leur sensibilité pour l'induction de tares héréditaires, elles sont également relativement insensibles à la diminution de la fécondité. Les expérimentations animales semblent montrer que la fréquence de certaines aberrations chromosomiques et de tares dominantes, liées au sexe, augmente de façon directement linéaire avec la dose; l'augmentation serait moindre en ce qui concerne les maladies "irrégulièrement héréditaires » les plus communes, notamment pendant les deux premières générations. Quand le corps entier est exposé de façon uniforme, on a estimé que le détriment héréditaire total est probablement moindre que celui dû aux lésions somatiques chez les individus irradiés. Dans l'optique de la radioprotection et en tenant compte de la proportion des expositions qui sont probablement significatives au point de vue génétique, on a estimé 
à environ $4 \times 10^{-3} \mathrm{~Sv}^{-1}$ le risque de dommages héréditaires sérieux pendant les deux premières générations suivant l'irradiation de l'un ou l'autre des parents, avec un dommage supplémentaire du même ordre de grandeur pour toutes les générations ultérieures $\left(^{1}\right)$.

Alors que le sein pendant la période d'activité génitale de la femme peut être l'un des tissus les plus radiosensibles, en effectuant une moyenne sur tous les âges et pour les deux sexes, on a été conduit, dans l'optique de la radioprotection, à une estimation du risque d'induction de cancer d'environ $2,5 \times 10^{-3} \mathrm{~Sv}^{-1}$.

Pour la moelle rouge des os, le risque principal est l'induction de leucémie. A partir des informations disponibles et dans une optique de radioprotection, la Commission a retenu pour ce risque la valeur de $2 \times 10^{-3} \mathrm{~Sv}^{-1}$.

On sait que l'irradiation externe du tissu pulmonaire peut induire, chez l'homme, un cancer du poumon. On estime, à l'heure actuelle, que ce risque est à peu près le même, par unité de dose, que le risque de leucémie dû à l'irradiation de la moelle osseuse.

Par ailleurs, la sensibilité de la thyroïde à l'induction de cancer par les rayonnements semble plus grande que celle de la moelle rouge des os à l'induction de leucémie. Cependant, la mortalité par cancer de la thyroïde est notablement plus faible que la mortalité par leucémie; le risque global de mortalité retenu par la Commission pour le cancer de la thyroïde est de $5 \times 10^{-4} \mathrm{~Sv}^{-1}$.

L'étude, par la Commission de la sensibilité des cellules du tissu osseux à l'induction de cancer par les rayonnements (cellules endostéales et cellules épithéliales à la surface de l'os) a montré qu'elle était très inférieure à celle du sein, de la moelle rouge, du poumon et de la thyroïde. Pour la radioprotection, on a également retenu pour la mortalité par tumeur osseuse un risque de $5 \times 10^{-4} \mathrm{~Sv}^{-1}$.

Outre les tissus énumérés dans le tableau, il en existe d'autres pour lesquels on sait que les rayonnements sont également cancérigènes à des doses modérées. Pour ceux-ci cependant, nous ne pouvons pas encore estimer le risque par unité de dose, bien qu'il soit probablement faible en comparaison des risques indiqués dans le tableau. De même, il y a des tissus tels que le muscle et le tissu adipeux pour lesquels on a peu de preuves d'induction de tumeur à des doses modérées. En considérant l'ensemble des tumeurs malignes radioinduites par rapport au risque de leucémie dans les mêmes conditions d'irradiation, il semble probable que la majorité survienne dans les tissus pour lesquels on a pu obtenir les risques approximatifs qui figurent au tableau ci-dessus. Sur cette base, on a estimé que les risques conjugués de tumeur maligne pour les autres tissus non spécifiés ne dépassent probablement pas $5 \times 10^{-3} \mathrm{~Sv}^{-1}$; on a, de plus, supposé qu'aucun tissu isolé ne pouvait compter pour plus du cinquième de cette valeur.

( ${ }^{1}$ Sv est l'abréviation de Sievert, unité d'équivalent de dose (1 Sv $\left.=100 \mathrm{rem}\right)$. 
A la réflexion, il est clair que certaines des valeurs du risque par unité de dose dépendent de l'âge et du sexe. Cependant, à la lumière des informations dont elle dispose, la Commission a conclu que, pour la majeure partie de la vie, les variations du risque en fonction du sexe et de l'âge par rapport aux valeurs moyennes indiquées dans le tableau ne sont pas considérables. De ce fait, bien que la distribution par classe d'âge de la population dans son ensemble diffère de celle des travailleurs professionnellement exposés aux rayonnements ionisants en sorte que les risques ne sont pas tout à fait les mêmes dans les deux groupes, les risques relatifs sont peu différents. La Commission n'a pas encore trouvé de différences suffisantes qui justifient un tableau distinct et une autre série de facteurs de pondération, $w_{i}$, pour limiter l'exposition des individus du public. C'est à partir de considérations de cette sorte et dans l'optique de la protection des individus que la Commission a conclu que le risque global de mortalité du fait de cancer radio-induit, à la suite d'une irradiation uniforme de l'organisme entier, est d'environ $10^{-2} \mathrm{~Sv}^{-1}$ en moyenne pour les deux sexes et pour tous les âges. Il faut ajouter à cette valeur le risque moyen de tares héréditaires graves apparaissant dans les deux premières générations, soit environ $4 \times 10^{-3} \mathrm{~Sv}^{-1}\left({ }^{2}\right)$.

Les limites d'équivalent de dose précédemment recommandées par la Commission sont en vigueur depuis vingt ans, sinon davantage. Il n'existe aucune preuve que le système de limitation de dose recommandé par la Commission n'ait pas atteint son but, qui est d'assurer un niveau convenable de sécurité.

Cependant, disposant maintenant de valeurs du risque par unité de dose, il est apparu souhaitable à la Commission de réexaminer ces limites d'équivalent de dose, notamment pour voir si des modifications des niveaux étaient indiquées.

Pour le proche avenir, la Commission considère qu'une méthode valable pour juger du caractère acceptable d'un niveau de risque dans les travaux sous rayonnements est de comparer ce risque avec ceux encourus dans d'autres professions connues pour présenter un haut niveau de sécurité. A partir des informations disponibles, le Groupe de Travail de la Commission sur les limites de dose a considéré comme «industries à haut niveau de sécurité » celles caractérisées par un taux annuel moyen de mortalité professionnelle d'environ $10^{-4}$ (100 par million de travailleurs). Sur cette base, la Commission a choisi comme critère provisoire la valeur de 50 décès par million et par an comme acceptable pour l'exposition professionnelle; réparti sur une durée de vie professionnelle de 40 ans, ce "risque nominal " dû à l'exposition professionnelle correspondrait à un risque total sur la durée de vie de $2 \times 10^{-3}$ [11]. Dans le cas d'une exposition uniforme de l'organisme entier auquel s'applique la limite d'équivalent de dose annuelle de $50 \mathrm{mSv}$ ( $5 \mathrm{rem}$ ), il s'avère que la distribution des équivalents de dose annuels est en gros log-normale, la moyenne arithmétique étant en général inférieure à $5 \mathrm{mSv}(0,5 \mathrm{rem})$ et très peu de valeurs approchant la limite. Ainsi, avec un

$\left(^{2}\right)$ Les valeurs du tableau (moins les effets génétiques) totalisent environ $1,3 \cdot 10^{-2} \mathrm{~Sv}^{-1}$.

voL. $13-\mathrm{N}^{\circ} 4$ 
risque global de $1,7 \times 10^{-2} \mathrm{~Sv}^{-1}$, on voit que le risque annuel moyen pour l'exposition professionnelle $\left(\sim 0,9 \times 10^{-4}\right)$ est comparable au risque moyen de décès dans les autres industries sûres. Il apparaît donc que la limite de dose fondamentale demeure raisonnable.

Les recommandations de la Commission visent non seulement à limiter à un niveau acceptable (que nous avons estimé être $10^{-4}$ par an) la probabilité d'apparition des effets stochastiques, mais également à exclure les effets non stochastiques. A la lumière des connaissances actuelles, la Commission estime que les effets non stochastiques seront prévenus en appliquant une limite d'équivalent de dose de $0,5 \mathrm{~Sv}$ (50 rem) par an pour tous les tissus, à l'exception du cristallin pour lequel elle recommande une limite annuelle de 0,3 Sv (30 rem). Ces limites sont destinées à établir une contrainte pour toute exposition qui satisfait par ailleurs la limitation des effets stochastiques.

Pour les effets stochastiques, puisqu'on s'est affranchi du concept d'organe critique, les limites de dose recommandées par la Commission sont basées désormais sur le principe que le risque doit être identique, que l'organisme entier soit uniformément irradié ou que l'irradiation n'intéresse que certains organes. On satisfait à cette condition si

$$
\sum_{i} w_{i} H_{i} \leqq H_{w b, L}
$$

où $w_{i}$ est un facteur de pondération représentant le rapport du risque stochastique résultant de l'irradiation du tissu $i$, au risque total dans le cas d'irradiation uniforme de l'organisme entier,

$H_{i}$ est l'équivalent de dose annuel dans le tissu $i$, et

$H_{w b, L}$ est la limite d'équivalent de dose annuel pour une irradiation uniforme du corps entier, soit $50 \mathrm{mSv}$ ( $5 \mathrm{rem})$.

Les valeurs de $w_{i}$ doivent servir de guide lors du calcul des limites dérivées et secondaires; le Comité 2 de la C.I.P.R. les a utilisées dans son nouveau. rapport concernant l'évaluation des limites annuelles d'incorporation des radionucléides, qui tiennent compte de l'équivalent de dose dans chaque tissu. Lorsqu'il y a, à la fois, exposition interne et externe, la limitation de dose recommandée par la Commission pour les effets stochastiques ne sera pas dépassée si

$$
\left(H_{I} / H_{w b, L}\right)+\sum_{j}\left(I_{j} / I_{j, L}\right) \leqq 1
$$

où $H_{I}$ est l'index d'équivalent de dose annuel, tel qu'il est défini par I'I.C.R.U. [16];

$I_{j}$ est l'incorporation annuelle du radionucléide $j$;

$I_{j, L}$ est la limite annuelle d'absorption du radionucléide $j$.

Dans ses nouvelles recommandations, la Commission ne propose pas de limites de dose pour les populations. Dans les précédentes, elle suggérait une limite de dose génétique, tout en exprimant des réserves sur la possibilité d'appliquer de façon générale à des populations des limites de dose parti- 
culières [7]. Il est clair, maintenant, que le niveau précédemment suggéré « à titre d'allocation » - 5 rem en 30 ans - ne peut être atteint; il est, de même, très improbable que les autorités nationales responsables puissent autoriser qu'un équivalent de dose annuel atteigne ne serait-ce qu'une petite fraction de la limite suggérée alors. Étant donné qu'on pourrait considérer que le maintien de l'ancienne limite permettrait d'une part de justifier une exposition de la population qui ne serait ni nécessaire ni vraisemblable et d'autre part d'accepter un risque plus élevé que ne le justifient les perspectives actuelles ou futures, la Commission - je le répète - ne propose pas, pour le moment, de limites de dose pour les populations. Elle souhaite plutôt souligner que toute contribution d'origine artificielle à l'exposition de la population doit être justifiée par ses avantages, Ainsi, la limite pour l'irradiation d'une population dans son ensemble sera le total atteint par la somme des contributions minimales nécessaires, compte tenu des risques et des avantages correspondants. Dans une telle analyse coût-avantage, pour une pratique donnée, par exemple, on peut prendre comme mesure directe du détriment correspondant l'équivalent de dose collectif à condition que cette dose soit limitée à la population qui en tirera bénéfice. Si l'exposition concerne d'autres populations, alors l'équivalent de dose collectif total devra demeurer inférieur à celui qu'on aurait appliqué si l'analyse coût-avantage avait été limitée à la population bénéficiaire.

Cependant, bien que la Commission n'avance pas, pour le moment, de limites de dose pour les populations, elle propose, comme par le passé, des limites d'équivalent de dose pour les membres individuels du public. Lorsqu'elle a abordé ce problème, la Commission a commencé par considérer l'ordre de grandeur des risques acceptés dans la vie courante par chaque citoyen. Après un examen de la situation, il est apparu à la Commission que, lorsqu'il n'était pas possible de réduire ces risques par un effort individuel ou de les éliminer totalement, leur acceptation était basée pour l'essentiel, soit sur les avantages dont on ne bénéficierait pas autrement, soit sur l'estimation implicite que le risque est négligeable. Le risque dans les transports en commun en est un exemple.

Après un examen des informations quantitatives actuelles, la Commission a conclu que le niveau d'acceptablilité d'un risque qui ne soulève que peu ou pas de problème dans le public était inférieur d'un ordre de grandeur par rapport aux risques professionnels. Sur cette base, la Commission a conclu qu'un risque annuel d'environ $10^{-6}-10^{-5}$ pouvait être acceptable pour tout membre individuel du public. Avec une valeur pour le risque total de $10^{-2} \mathrm{~Sv}^{-1}$, un risque annuel moyen de $5 \times 10^{-6}$ (pour un membre du public) correspondra à une exposition annuelle moyenne de $0,5 \mathrm{mSv}(50 \mathrm{mrem})$ ou à une limite de dose de $5 \mathrm{mSv}$ par an, c'est-à-dire à une limite de dose de $0,5 \mathrm{rem}$ par an pour une exposition uniforme de l'organisme entier dans le groupe critique. Pour des besoins d'organisation, la Commission a conclu qu'il conviendra de continuer à estimer les doses en fonction d'une limite d'équivalent de dose de $5 \mathrm{mSv}$ par an. Avec une limite ainsi définie et sur la base de la distribution de fréquence des doses à l'intérieur des populations soumises $\mathrm{au}$ risque, on peut s'attendre à ce que l'exposition moyenne soit inférieure 
à $0,5 \mathrm{mSv}$ par an. Afin de prévenir tout effet non-stochastique, il est également recommandé d'appliquer une limite d'équivalent de dose de $50 \mathrm{mSv}$ par an à laquelle il ne saurait être dérogé. Il est certain que cette valeur est très inférieure (en fait $1 / 10^{\mathrm{e}}$ ) à la valeur recommandée pour la prévention des effets nonstochastiques dans le cas de l'exposition professionnelle. Le but est de faire en sorte qu'une plus longue période d'exposition et les difficultés pratiques du contrôle de l'exposition totale des membres du public n'entraînent pas de dépassement des seuils.

En résumé, les fondements des recommandations de la Commission sont passées de l'expérience des rayons $\mathrm{X}$ à une philosophie du risque - le risque, dans chaque cas, se justifiant par les avantages qui en découlent. Sur cette base nouvelle, la limite de 5 rem par an pour une exposition uniforme du corps entier recommandée précédemment apparaît toujours raisonnable à la lumière des risques encourus dans les autres professions. Le concept d'organe critique a été abandonné; à sa place est apparu celui de l' " équi-détriment », c'est-à-dire que la limite de dose annuelle admise pour un organe isolé ou pour une combinaison quelconque d'organes ne doit pas dépasser le risque de 5 rem reçu uniformément par l'organisme.

\section{BIBLIOGRAPHIE}

[1] Grubbé E. H. X-ray Treatment, its origin, birth and early history. St. Paul, Minn., The Bruce Publishing Co., 1949.

[2] Roentgen W. C. Über eine Art von Strahlen (Vorläufige Mitteilung). Sitzungsberichte der Physik.-Med. Ges., Wurzburg, 1895, 132 (A translation into the English language may be found in Wilhelm Conrad Roentgen and the early history of Roentgen rays, by Otto Glasser Springfield, Ch. C. Thomas, 1934).

[3] Becquerel H. Sur les radiations émises par phosphorescence. C. R. Acad. Sc., 1896 122, 420. Sur les radiations invisibles émises par les corps phosphorescents, ibid., p 501; Sur quelques propriétés nouvelles des radiations émises par divers corps phosphorescents, ibid. p. 559.

[4] Ledoux-Lebard R. Le Cancer des radiologistes. Paris Méd., 1922, 45, 299.

[5] Recommendations of the International Commission on Radiological Protection, revised by the International Commission on Radiological Protection, London, 1950. 6th International Congress of Radiology, London.

[6] Recommendations of the International Commission on Radiological Protection, revised Dec. 1, 1954. Br. J. Radiol., 1955, Suppl. 6.

[7] Recommendations of the International Commission on Radiological Protection, adopted Sept. 9, 1958. I.C.R.P. Publication 1. Oxford, Pergamon Press, 1959.

[8] Recommendations of the International Commission on Radiological Protection, as amended 1959 and revised 1962, I.C.R.P. Publication 6. Oxford, Pergamon Press, 1964.

[9] Recommendations of the International Commission on Radiological Protection, adopted Sept. 17, 1965, I.C.R.P. Publication 9. Oxford, Pergamon Press, 1966.

[10] TAYLOR L. S. Radiation exposure as a reasonable calculated risk. Health Physics, 1958, 1,62 . 
[11] Registrar General's Decennial Supplement. England \& Wales, Occupational mortality. London, H. M. Stationery Office, 1961.

[12] Doll R., Fisher R. E. W., Garmon E. J., Gunn W., Hughes G. O., Tyrer F. H. and WILSON W. Mortality of gasworkers with special reference to cancers of the lung and bladder, chronic bronchitis, and pneumoconiosis. Br. J. Industr. Med., 1965, 22, 1.

[13] Acheson E. D., Cowdell R. H., Hadfield E., and Macbeth R. G. Nasal cancer in woodworkers in the furniture industry. Br. Med. J., 1968, 587.

[14] Acheson E. D., Cowdell R. H. and Jolles B. Nasal cancer in the Northamptonshire boot and shoe industry. Br. Med. J., 1970, 385.

[15] Bäuml A., Kurez I., Millmann L. and Wachsmann F. Die Strahlenbelastung von in der Röntgendiagnostik arbeitenden Personen. Fortschr. Röntgenstr., 1974, 120, 84.

[16] International Commission on Radiation Units and Measurements. Conceptual basis for the determination of dose equivalent, ICRU Report 25. Washington, ICRU, 1976.

\section{REMERCIEMENTS}

La rédaction remercie vivement $\mathrm{M}$. Dardenne et $\mathrm{M}^{\mathrm{me}}$ Beau pour leur contribution à la traduction de ce texte. 